\title{
Outcome of Percutaneous Radiofrequency Thoracic Sympathectomy for Palmar Hyperhidrosis
}

\author{
Flávio Ramalho Romero, $M D, P h D,{ }^{*}$ Daniele Cristina Cataneo, $M D, P h D,{ }^{\dagger}$ and \\ Antonio José Maria Cataneo, $M D, P h D^{\dagger}$
}

The impact of upper thoracic percutaneous sympathectomy with radiofrequency on the quality of life (QOL) of patients with palmar hyperhidrosis was evaluated. Thirty-six patients with palmar hyperhidrosis were selected for a prospective observational study. Treatment consisted of percutaneous radiofrequency thoracic sympathectomy of T3 and T4 ganglions in all cases. QOL questionnaires were applied preoperatively, on the 1st postoperative (PO) day, and on the 30th, 90th, 180th, and 360th PO days. Furthermore, compensatory hyperhidrosis (HDSSc) scale measures were used simultaneously, in order to evaluate the rate and frequency of this side effect. The QOL questionnaire evaluation showed preoperative values of $83.94 \pm 4.74$ (meaning poor quality of life), decreasing to $24.61 \pm 2.86$ on the 1st PO day, $25.14 \pm 3.12$ on the 30th PO day, $31.28 \pm 4.42$ on the 90th $\mathrm{PO}$ day, $32.97 \pm 4.54$ on the 180th PO day, and $33.94 \pm 4.6$ on the 360th PO day (all postoperative results with values below 35 were considered optimal). Compensatory hyperhidrosis (HDSSc) scale values were $1.14 \pm 0.35$ on the 1st $\mathrm{PO}$ day, $1.42 \pm 0.55$ on the 30th PO day, $1.83 \pm 0.85$ on the 90th PO day, $1.92 \pm 0.91$ on the 180th PO day, and $1.92 \pm 0.91$ on the 360th PO day (meaning that hyperhidrosis was mainly unnoticed). Patients' subjective satisfaction was considered very good and the majority of patients would recommend the treatment procedure. Percutaneous radiofrequency thoracic sympathectomy had a positive impact on the quality of life of patients with palmar hyperhidrosis, compared to the surgical treatment, with a low rate and intensity of HDSSc and without other complications.

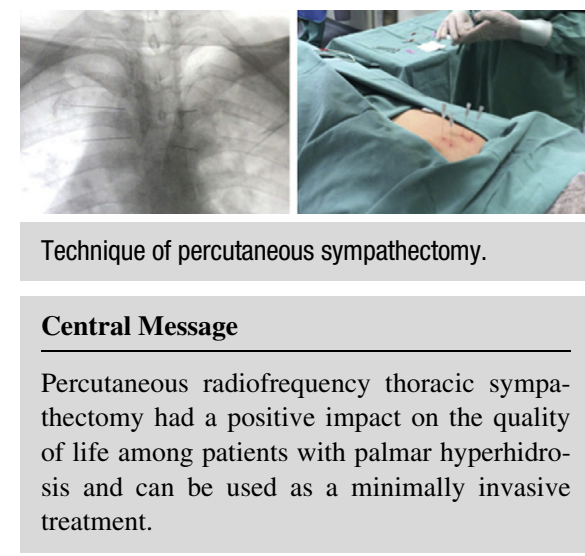

Perspective Statement

Percutaneous sympathectomy using radiofrequency techniques has been used as an alternative to surgical procedure for twenty years, in a few centers of hyperhidrosis around the world. There are a few comparative studies showing the superiority of surgical approach over the percutaneous technique with regards to sweat control. Our study demonstrated the equality between the results of techniques.

Semin Thoracic Surg 30:362-366 @ 2018 Elsevier Inc. All rights reserved.

Keywords: Palmar hyperhidrosis, Percutaneous sympathectomy, Radiofrequency, Compensatory hyperhidrosis

Abbreviations: HDSSc, compensatory hyperhidrosis; DLQI, Dermatol-
ogy Life Quality Index; DLQI, Hyperhidrosis Disease Severity Scale;
QOL, quality of life
${ }^{* B}$ Botucatu School of Medicine, São Paulo State University - UNESP,
Brazil.
'Division of Thoracic Surgery. Botucatu School of Medicine, São Paulo
State University - UNESP, Brazil.
Disclosures: none
Funding statement: none
Conflicts of interest: none

Address reprint requests to Daniele Cristina Cataneo, Thoracic Surgery Division, Department of Surgery, São Paulo State University - UNESP, Distrito de Rubião Júnior s/n, Botucatu, Brazil. E-mail:

dcataneo@fmb.unesp.br

\section{INTRODUCTION}

Sweating is a physiological process that increases during physical activity and stress. Hyperhidrosis is a disorder of excessive sweating in certain regions of the body, such as the palms, soles, face, and axillae, ${ }^{1-3}$ with incidence ranging from 0.6 to $2.8 \%,{ }^{4}$ causing significant discomfort for patients and affecting patient quality of life (QOL). ${ }^{1,5-7}$ A possible etiology is attributed to functional impairment in the sympathetic nervous system, but the exact cause is still unknown.,9

Treatment options include nonsurgical (eg topical agents, iontophoresis, botulinum toxin A, tricyclic antidepressants and anticholinergic agents) ${ }^{10-12}$ and surgical modalities (eg liposuction and endoscopic sympathectomies $)^{1,13-15}$. Despite 
many treatment options, severe hyperhidrosis has a higher control rate through surgical sympathectomy; however, some complications have been described. ${ }^{16-20}$

Thoracic sympathectomy can be performed not only by surgical intervention but also with percutaneous sympathetic neurolysis or radiofrequency ablation. Wilkinson was the first to describe a percutaneous technique for radiofrequency thermocoagulation, for untreatable pain in the upper extremities. ${ }^{21,22}$ After some years, other authors began to use this procedure to treat patients with palmar hyperhidrosis. ${ }^{23-25}$ Radiofrequency therapy is a minimally invasive modality that uses electromagnetic energy near the nerve tissue. ${ }^{26-28}$ Despite previous results reports, none evaluated QOL as a systematic outcome.

Our purpose was to evaluate the impact of upper thoracic percutaneous sympathectomy with radiofrequency on the QOL in patients with palmar hyperhidrosis, with a long-time follow-up, using QOL questionnaires, scales, and measurements of palmar temperature both before and after the procedure.

\section{METHODS}

Patients diagnosed with palmar hyperhidrosis and treated at São Paulo State University Hospital between January 2013 and March 2014 were selected for a prospective observational cohort study. The inclusion criteria were age, between 18 and 45 years, and diagnosis of moderate or severe palmar hyperhidrosis. The exclusion criteria were secondary causes of hyperhidrosis (endocrine, neurological, and oncological conditions, infection, drug use and/or abuse), body mass index (BMI) greater than $28 \mathrm{~kg} / \mathrm{m}^{2}$, concomitant or recent acute myocardial infarction, recent surgery (less than 30 days), and/or clinical or laboratory evidence of acute or chronic systemic infection, renal or hepatic diseases, pregnancy, drug and alcohol abuse, and other conditions that affect life expectancy.

The treatment consisted of percutaneous thoracic sympathectomy of T3 and T4 ganglions, for all patients. A digital palmar thermometer was employed to evaluate differences in palmar temperature during the procedure (before and right after the procedure) and after one year of follow-up. ${ }^{23,29-31}$

Preoperative and postoperative (1st day, 30th day, 90th day, 180th day, and 360th day after the procedure) evaluation was performed using the Campos QOL questionnaire ${ }^{1}$ and the Dermatology Life Quality Index (DLQI). ${ }^{32}$ The questionnaire assesses the QOL of individuals with hyperhidrosis before and after treatment. It presents values from 0 to 100, where 100 before treatment means very poor QOL and in the posttreatment means that QOL was much worse. The DLQI is a validated tool that measures the degree of change in QOL after treatment of dermatological disease that consists of 10 questions with five possible responses: very much, a lot, mild, a little, and not at all (corresponding scores from 5 to 1 -the higher the score, the greater the impairment in QOL). During the follow-up (one year later), the patients were submitted to a subjective satisfaction questionnaire that consists of questions that have three or four pre-established answers: patient satisfaction (very satisfied, satisfied, not satisfied), status of the hand (dry, fairly dry, not dry), and the degree of compensatory hyperhidrosis (HDSSc) (absent, mild, moderate, severe) that is the most frequent complaint. Other questions referred to the increase of QOL and whether the patient would recommend the procedure to a friend, requiring the answer yes or no. The Hyperhidrosis Disease Severity Scale (HDSS) was used to evaluate the disease (before and after the procedure) and also to evaluate the HDSSc after the procedure. The scale analyzes the impact of sweating on daily activities, ranging from 1 (never noticed) to 4 (unbearable). ${ }^{33}$

The surgical protocol was the same in every patient and was performed by the same neurosurgeon (FRR). The patients were monitored (cardiac and oxygen saturation), draped, and placed in the prone position in a surgical room, under local anesthesia, to position the 5-mm active cannula of the radiofrequency device to the right T3 sympathetic ganglion. The cannula had to be pointed parallel to the ganglion, to apply the physical principles of radiofrequency and optimize the thermic lesion to the target, ${ }^{26-28}$ and the confirmation of the positioning was made under continuous fluoroscopic guidance. Then, some tests were performed using the electrode of the radiofrequency device. The impedance was set between 200 and $400 \Omega$, paresthesia was observed with $50-\mathrm{Hz}$ sensory stimulation at $0.3-0.5 \mathrm{~V}$ and no motor contractions were observed with $2-\mathrm{Hz}$ motor stimulation at $1.3-1.5 \mathrm{~V}$. After the neurophysiological testing, $2 \mathrm{ml}$ of $2 \%$ lydocaine was applied through the cannula (Figure 1) and the patient was mildly sedated (midazolam) to start radiofrequency thermal coagulation $\left(80^{\circ} \mathrm{C}\right.$ for 90 seconds). The same sequence was performed on the left T3 ganglion and bilaterally on T4 ganglions. Patients were monitored for potential complications for 3 hours following the procedure and discharged on the same day.

The institutional Research Ethics Board approved this study; all patients signed their informed consent before the procedure. Descriptive statistics were presented as frequency, percentage, mean, and standard deviation. Data were represented by mean and standard deviation (quantitative) for each moment. To compare the different moments of evaluation, normality tests of Shapiro-Wilks and Kolmogory-Smirnov with the correction of Cramer-von Milses and AndersonDarling were applied. The comparison between means was done by repeated measurements using Anova, followed by the Duncan multiple-comparison test considering pre-operative as a baseline in the case of normality. Otherwise, the same analysis was used adjusting a gamma distribution followed by the Wald multiple-comparison test. A $P$ value of $<0.05$ was considered significant. Statistical analysis was performed with SAS 9.3 for Windows.

\section{RESULTS}

Between March 2013 and January 2014, 36 patients with palmar hyperhidrosis were selected according to the inclusion and exclusion criteria. The majority of patients were female (26 patients-73\%), with a mean age of $23.6 \pm 5.2$ years 


\section{THORACIC - THORACIC SYMPATHECTOMY FOR PALMAR HYPERHIDROSIS}
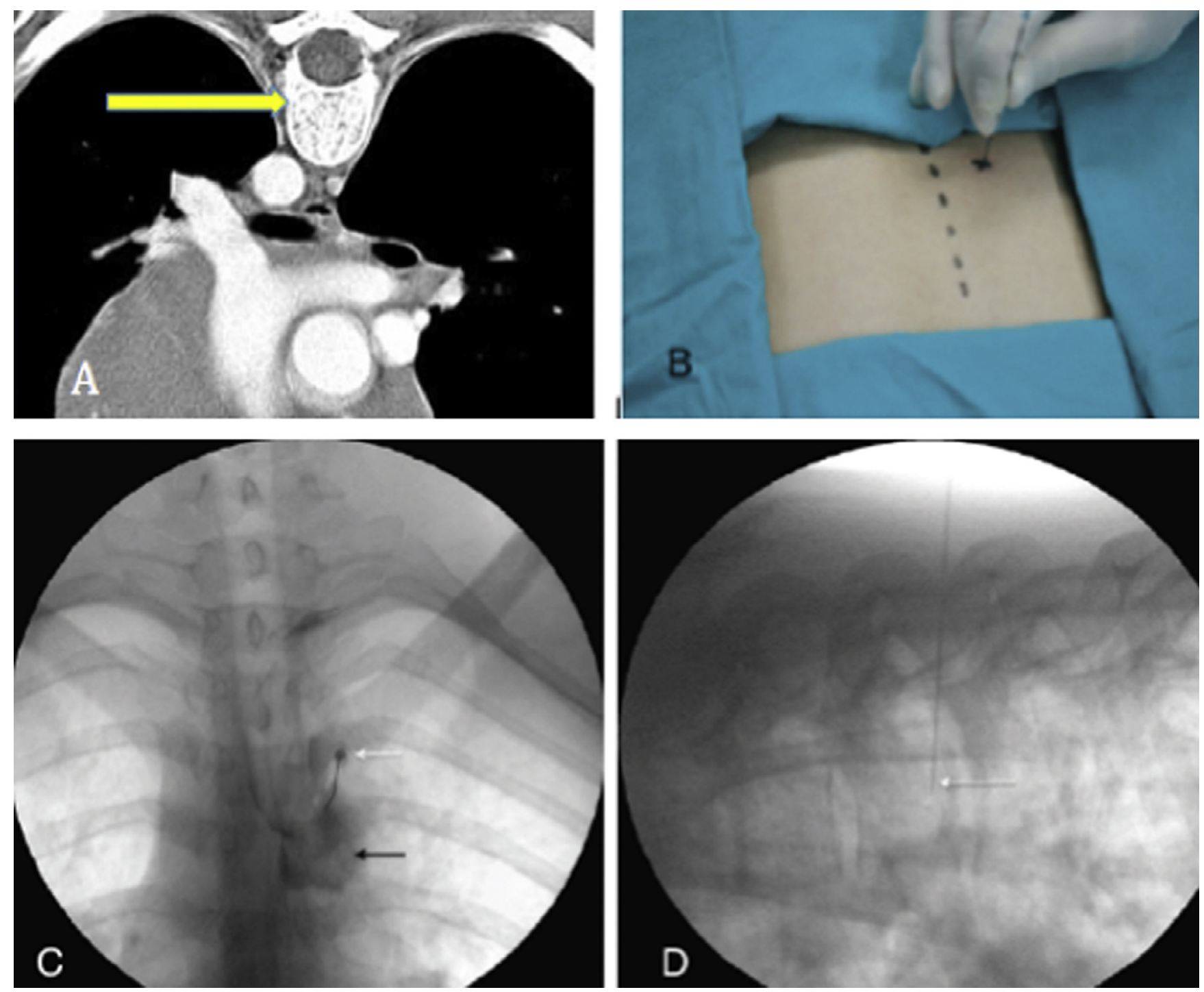

Figure 1. Example of percutaneous sympathectomy. A. CT scan-the arrow displays the location of the ganglion. B. Positioning the cannula through the patient's skin. C. Antero-posterior intraoperative fluoroscopic view-exhibits the correct electrode position. D. Lateral intraoperative fluoroscopic view - exhibits the cannula position (white arrow).

(range from 18 to 42 years). According to their skin color, $75 \%$ were white (27 patients) and the remaining were black. Every patient had received previous clinical treatment: topical substances were used in $89 \%$, botulinum toxin $\mathrm{A}$ in $11 \%$, and anticholinergic agents in $14 \%$. Besides palmar hyperhidrosis, $34 \%$ of patients had concomitant axillar sweating, and $89 \%$ had concomitant plantar hyperhidrosis. No cephalic hyperhidrosis was observed.

Serious complications were not observed during or after the procedure (eg cardiac arrest, permanent bradycardia, Horner syndrome, hemothorax, pneumothorax, atelectasis, motor deficit, pulmonary edema) and pain in the first hours was the main complaint, solved with ordinary painkillers. Palmar temperature decreased at least $1^{\circ} \mathrm{C}$ during the procedure (average $34.1^{\circ} \mathrm{C}$ to $31.8^{\circ} \mathrm{C}$ ) and after 12 months (mean $33.3^{\circ} \mathrm{C}$ ) (Table 1 ).
The HDSS and DLQI had a great improvement (Table 1), as well as the Campos QOL questionnaire (Table 1). Results of the HDSSc evaluation, measured by the HDSS, are also summarized in Table 1.

The patients' subjective satisfaction was high (Table 2); $92 \%$ reported increase in QOL and 90\% of them (32 patients) would recommend the procedure.

\section{DISCUSSION}

Radiofrequency percutaneous sympathectomy has been performed as an alternative to surgical procedure to treat hyperhidrosis for 20 years, in a few centers around the world. ${ }^{23-25}$ There are a few comparative studies demonstrating the superiority of a surgical approach over the radiofrequency technique with regards to sweat control. Our data, when analyzing QOL 


\section{THORACIC - THORACIC SYMPATHECTOMY FOR PALMAR HYPERHIDROSIS}

Table 1. Results of Scales, QOL Questionnaires, and Measurement of Temperature

\begin{tabular}{lrcccccc}
\hline Follow- up & Preoperative & \multicolumn{1}{c}{ First Day } & \multicolumn{1}{c}{ 1 Month } & \multicolumn{1}{c}{3 Months } & 6 Months & 1 Year & $P$ value \\
\hline HDSS $^{1}$ & $3.64 \pm 0.54$ & $1 \pm 0$ & $1 \pm 0$ & $1.08 \pm 0.28$ & $1.61 \pm 0.64$ & $1.64 \pm 0.64$ & $<0.0001$ \\
DLQ $^{2}$ & $39.58 \pm 4.46$ & $10.58 \pm 0.87$ & $10.56 \pm 0.81$ & $11.83 \pm 2.75$ & $12.75 \pm 2.78$ & $13.17 \pm 3.26$ & $<0.0001$ \\
QOL $^{3}$ & $83.94 \pm 4.74$ & $24.61 \pm 2.86$ & $25.14 \pm 3.12$ & $31.28 \pm 4.42$ & $32.97 \pm 4.54$ & $33.94 \pm 4.6$ & $<0.0001$ \\
HDSC $^{\#}$ & - & $1.14 \pm 0.35$ & $1.42 \pm 0.55$ & $1.83 \pm 0.85$ & $1.92 \pm 0.91$ & $1.92 \pm 0.91$ & $<0.0001$ \\
Temperature* $^{*}$ & $34.09 \pm 0.26$ & $31.83 \pm 0.57$ & - & - & - & $33.30 \pm 0.44$ & $<0.0001$ \\
\hline
\end{tabular}

${ }^{1}$ Hyperhidrosis Disease Severity Scale (HDSS).

${ }^{2}$ Dermatology Life Quality Index (DLQI).

${ }^{3}$ Campos QOL questionnaire [1].

\#Compensatory hyperhidrosis (HDSSc) was evaluated on the first postoperative day and the significant difference was between that moment and the other postoperative days.

*Temperature was measured only before the procedure, on the first postoperative day, and one year later and the significant difference was between the first moment and the other postoperative days.

questionnaires and patients' subjective satisfaction, were slightly superior compared to other published studies. Purtuloglu et $\mathrm{al}^{25}$ had a success rate of $75 \%$ in a percutaneous technique and $95 \%$ in a thoracoscopic approach. We observed a $92 \%$ rate of success in our patients, considering the subjective satisfaction and improvement of QOL. This high success rate could be attributed to our choice of target for ablation (T3 and T4 ganglions) and strict application of radiofrequency's physical principle that consists in the positioning of the cannula tip parallel to the ganglion. ${ }^{26-28}$

The choice of $\mathrm{T} 3$ and $\mathrm{T} 4$ ganglions, compared to that of other studies which selected T2 and T3 or only $\mathrm{T}^{23-25}$ (patients complain about moderate or severe compensatory or wet hands), could explain our high success rate regarding hyperhidrosis long-term control. ${ }^{17-19}, 23-25$ Some studies demonstrated that dryness in the hands is better reached with two levels of ablation and some studies attributed the intensity of HDSSc to more than one level and both are related to satisfaction. ${ }^{13,19}$ Similarly, some studies do not attribute the number of levels of ablation to HDSSc. ${ }^{20}$ We believe that the cause of both is multifactorial. Although the literature is inconclusive, we don't need to reach higher ablation levels to obtain dry hands, it being possible to reach good results simultaneously avoiding HDSSc.

Table 2. Results of Subjective Satisfaction Questionnaire

\begin{tabular}{clllll}
\hline & \multicolumn{1}{l}{$\begin{array}{l}\text { Very } \\
\text { Satisfied }\end{array}$} & Satisfied & $\begin{array}{l}\text { Not } \\
\text { Satisfied }\end{array}$ & Total \\
\cline { 2 - 6 } Patient satisfaction & 26 & 7 & 3 & 36 \\
& $72 \%$ & $20 \%$ & $8 \%$ & $100 \%$ \\
& Dry & Fairly dry & Not dry & Total \\
\cline { 2 - 6 } Status of the hand & 32 & 2 & 2 & 36 \\
& $89 \%$ & $5.5 \%$ & $5.5 \%$ & $100 \%$ \\
\cline { 2 - 6 } $\begin{array}{c}\text { Compensatory } \\
\text { hyperhidrosis }\end{array}$ & 28 & 4 & 3 & 1 & 36 \\
& Absent & Mild & Moderate & Severe & Total \\
\hline & $78 \%$ & $11 \%$ & $8 \%$ & $3 \%$ & $100 \%$ \\
\hline
\end{tabular}

Authors advocate the theory that recurrence of sweat would be associated with another sympathetic ganglion adaptation to preserve the function of the ablated ganglion. ${ }^{19}$ On the other hand, the knowledge and application of physical radiofrequency principles, optimizing the heat wave and lesion area around the lateral portion of the cannula, and not in its extremity, increases the efficacy of the procedure. ${ }^{26-28}$ None of the papers found in the literature described and were concerned with these physical principles, and Purtuloglu et $\mathrm{al}^{24,25}$ had described the target in contact with the cannula's extremity.

Palmar temperature measurement was obtained as objective data to evaluate the procedure's efficacy (effectiveness of the nerve injury $)^{23,29}$ and the values decreased after the procedure. There are not many literature studies that employ palmar temperature measurement to evaluate the efficacy of the procedure for palmar hyperhidrosis ${ }^{29-31}$; however, these measurements are difficult to perform since they must take into account mainly the anesthesia and the temperature in the operating room. In our study, there was no anesthesia and we kept the room at a controlled temperature $26^{\circ} \mathrm{C}$ for at least 30 minutes, for acclimatization of the patient, and the temperature measurement was continuous during the procedure.

Our results using the radiofrequency technique showed a decrease of $1^{\circ} \mathrm{C}$ after the lesion in the sympathetic chain, which differs from other studies that showed a raise of at least $1^{\circ} \mathrm{C} .^{29-31}$ However, a recent study has demonstrated results similar to ours, with a decrease in the temperature especially at levels below T3, which could be attributed to a lower denervation at these levels due to the sympathetic anatomy. ${ }^{31}$

Many adverse effects are associated with thoracic sympathectomy ${ }^{1,16-20}$ but the main factor interfering in a patient's satisfaction is the HDSSc, with incidence ranging from 60 to $90 \% .{ }^{16-20}$ This theory could explain our higher patient satisfaction rate compared to the current literature data. Our data demonstrated that HDSSc was absent in $78 \%$ in 12 months and these results could be associated with our selected ganglions for the procedure (T3 and T4), since some studies have related a higher incidence of HDSSc in lesions above the T3 ganglion. ${ }^{18,19}$ 
A possible limitation is the fact that our study is not a randomized trial; there is no control group and no other technique for comparison. However, our study is a prospective observational study with a long follow-up time, and shows great and sustainable increase in patient QOL. As a result, thoracic percutaneous radiofrequency $\mathrm{T} 3$ and $\mathrm{T} 4$ ablation can be used as a safe and effective option in the treatment of palmar hyperhidrosis, being a minimally invasive procedure with satisfactory results, compared to surgical sympathectomy. Therefore, percutaneous radiofrequency thoracic sympathectomy had a positive impact on QOL among patients with palmar hyperhidrosis, with a low rate and intensity of HDSSc and without other complications.

\section{SUPPLEMENTARY MATERIAL}

The following is the supplementary data to this article:

\section{Percutaneous thoracic sympatectomy with radiofrequency}

\section{Hospital das Clínicas \\ São Paulo State University - Botucatu - Brazil}

Video S1. Technique of percutaneous sympathectomy.

\section{REFERENCES}

1. De Campos JRM, Kauffman P, Werebe EC, et al: Quality of life, before and after thoracic sympathectomy-report on 378 operated patients. Ann Thorac Surg 76:886-891, 2003

2. Hornberger J, Grimes K, Naumann M, et al: Multi-specialty working group on the recognition, diagnosis, and treatment of primary focal hyperhidrosis. Recognition, diagnosis, and treatment of primary focal hyperhidrosis. J Am Acad Dermatol 51:274-286, 2004

3. Wörle B, Rapprich S, Heckmann M: Definition and treatment of primary hyperhidrosis. JDDG 7:325-328, 2007

4. Strutton DR, Kowalski JW, Glaser DA, et al: US prevalence of hyperhidrosis and impact on individuals with axillary hyperhidrosis: results from a national survey. J Am Acad Dermatol 51:241-248, 2004

5. Kamudoni P, Mueller B, Halford J, et al: The impact of hyperhidrosis on patients' daily life and quality of life: a qualitative investigation. Health Qual Life Outcomes 15:121, 2017

6. Kouris A, Armyra K, Christodoulou C, et al: Quality of Life in Patients with Focal Hyperhidrosis before and after Treatment with Botulinum Toxin A. ISRN Dermatol 2014:308650

7. Cohen JL, Cohen G, Solish N, et al: Diagnosis, impact, and management of focal hyperhidrosis: treatment review including botulinum toxin therapy. Plast Surg Clin N Am 15:17-30, 2007

8. Vetrugno R, Liguori R, Cortelli P, et al: Sympathetic skin response: basic mechanisms and clinical applications. Clin Auton Res 13:256-257, 2003

9. Eisenhach JH, Atkinson JLD, Fealey RD: Hyperhidrosis: evolving therapies for a well-established phenomenon. Mayo Clin Proc 80:657-666, 2005

10. Togel B, Greve B, Raulin C: Current therapeutic strategies for hyperhidrosis: a review. Eur J Dermatol 12:219-223, 2002
11. Gee S, Yamauchi PS: Nonsurgical management of hyperhidrosis. Thorac Surg Clin 18:141-155, 2008

12. Murray CA, Cohen JL, Solish N: Treatment of focal hyperhidrosis. J Cutan Med Surg 11:67-77, 2007

13. Kopelman D, Hashmonai M, Schick C: The surgical treatment of hyperhidrosis. Ann Thorac Surg 93:1021-1022, 2012

14. Kim WO, Kil HK, Yoon KB, et al: Influence of T3 or T4 sympathectomy for palmar hyperhidrosis. Am J Surg 199:166-169, 2010

15. Raposio E, Caruana G: Video-assisted thoracic sympathicotomy for the treatment of palmar and axillary hyperhidrosis: a 17-year experience. Surg Laparosc Endosc Percutan Tech 25:417-419, 2015

16. Maillard H, Lecouflet M: Management of hyperhidrosis. Ann Dermatol Venereol 142:252-261, 2015

17. Rodríguez PM, Freixinet JL, Hussein M, et al: Side effects, complications and outcome of thoracoscopic sympathectomy for palmar and axillary hyperhidrosis in 406 patients. Eur J Cardiothorac Surg 34:514-519, 2008

18. Kopelman D, Hashmonai M: The correlation between the method of sympathetic ablation for palmar hyperhidrosis and the occurrence of compensatory hyperhidrosis: a review. World J Surg 32:2343-2356, 2008

19. Neumayer C, Zacherl J, Holak G, et al: Limited endoscopic thoracic sympathetic block for hyperhidrosis of the upper limb: reduction of compensatory sweating by clipping T4. Surg Endosc 18:152-156, 2004

20. Gunn TM, Davis DM, Speicher JE, et al: Expanded level of sympathetic chain removal does not increase incidence or severity of compensatory hyperhidrosis after endoscopic thoracic sympathectomy. J Thorac Cardiovasc Surg 148:2673-2676, 2014

21. Wilkinson HA: Percutaneous radiofrequency upper thoracic sympathectomy: a new technique. Neurosurgery 15:811-814, 1984

22. Wilkinson HA: Radiofrequency percutaneous upper-thoracic sympathectomy. Technique and review of indications. N Engl J Med 311:34-36, 1984

23. Garcia Franco CE, Perez-Cajaraville J, Guillen-Grima F, et al: Prospective study of percutaneous radiofrequency sympathicolysis in severe hyperhidrosis and facial blushing: efficacy and safety findings. Eur J Cardiothorac Surg 40:e146-e151, 2011

24. Purtuloglu T, Deniz S, Atım A, et al: A new target of percutaneous sympathic radiofrequency thermocoagulation for treatment of palmar hyperhidrosis: T4. Agri 25:36-40, 2013

25. Purtuloglu T, Atim A, Deniz S, et al: Effect of radiofrequency ablation and comparison with surgical sympathectomy in palmar hyperhidrosis. Eur J Cardiothorac Surg 43:e151-e154, 2013

26. Cosman Jr ER, Dolensky JR, Hoffman RA: Factors that affect radiofrequency heat lesion size. Pain Med 15:2020-2036, 2014

27. Cosman Jr ER, Cosman Sr ER: Electric and thermal field effects in tissue around radiofrequency electrodes. Pain Med 6:405-424, 2005

28. Cosman ER, Rittman WJ, Nashold BS, et al: Radiofrequency lesion generation and its effect on tissue impedance. Appl Neurophysiol 51: 230-242, 1988

29. Kao MC, Tsai JC, Lai DM, et al: Autonomic activities in hyperhidrosis patients before, during, and after endoscopic laser sympathicotomy. Neurosurgery 34:262-268, 1994

30. Li X, Tu YR, Lin M, et al: Minimizing endoscopic thoracic sympathicotomy for primary palmar hyperhidrosis: guided by palmar skin temperature and laser Doppler blood flow. Ann Thorac Surg 87:427-431, 2009

31. Liu Y, Li H, Zheng X, et al: Sympathicotomy for palmar hyperhidrosis: the Association between intraoperative palm temperature change and the curative effect. Ann Thorac Cardiovasc Surg 21:359-363, 2015

32. Finlay AY, Khan GK: Dermatology Life Quality Index (DLQI)—a simple prac tical measure for routine clinical use. Clin Exp Derrnatol 19:210-216, 1994

33. Solish N, Bertucci V, Dansereau A, et al: Canadian Hyperhidrosis Advisory Committee. A comprehensive approach to the recognition, diagnosis, and severity- based treatment of focal hyperhidrosis: recommendations of the Canadian Hyperhidrosis Advisory Committee. Dermatol Surg 33: 908-923, 2007 